\title{
INQUÉRITO DE CONHECIMENTO, ATITUDE E PRÁTICA: O AUTOCUIDADO E CONDUTAS DE PACIENTES EM TRATAMENTO PARA TUBERCULOSE
}

\section{KNOWLEDGE, ATTITUDE AND PRACTICE SURVEY: THE SELF- CARE AND CONDUCTS OF PATIENTS UNDER TREATMENT FOR TUBERCULOSIS}

Ana Caroline Cavalcante de Menezes ${ }^{1}$ Valdízia Mendes e Silva ${ }^{2}$ Jéssica Lins de Oliveira ${ }^{3}$ Mona Laura de Sousa Moraes ${ }^{4}$

Tânia Maria Ribeiro Monteiro de Figueiredo ${ }^{5}$

RESUMO: OBJETIVO: conhecer o perfil epidemiológico dos doentes em tratamento de tuberculose, bem como, seu conhecimento, atitude e prática frente à doença, avaliando aspectos como autocuidado e conduta. MÉTODOS: estudo transversal descritivo e quantitativo realizado através de um inquérito de Conhecimento, Atitude e Prática, no município de Campina Grande-PB. A população foi composta pelos pacientes diagnosticados com tuberculose entre setembro de 2017 a março de 2018. Os dados secundários foram obtidos por meio do banco de dados do Sistema de Informação de Agravos e Notificação disponibilizado pela Secretaria de Saúde do município de Campina Grande-PB. Os dados primários foram coletados por formulário e sua análise compreendeu os valores de distribuição de frequências absolutas e relativas, médias aritméticas e obtenção de indicadores epidemiológicos. Quanto aos conceitos de Conhecimento, Atitude e Prática, utilizou-se o referencial teórico-metodológico de Marinho et al. RESULTADOS: predominância do sexo masculino, faixa etária economicamente ativa, etnia/cor parda, nível de escolaridade relativamente baixo, $34,5 \%$ estavam desempregados e $31 \%$ exerciam trabalho

\footnotetext{
${ }^{1}$ Enfermeira, Mestranda em Saúde Pública (PPGSP-UEPB). E-mail: anacaroliinec@hotmail.com

2 Graduanda em Enfermagem pela Universidade Estadual da Paraíba (UEPB). E-mail: valdiziamendes@hotmail.com.

3 Graduanda em Enfermagem pela Universidade Estadual da Paraíba (UEPB). E-mail: jessicalins.05@gmail.com.

${ }^{4}$ Enfermeira, Mestre em Saúde Pública (PPGSP-UEPB). E-mail: monalauramoraes@gmail.com.

${ }^{5}$ Professora Doutora do Curso de Pós-Graduação Em Saúde Pública da Universidade Estadual daParaíba. E-mail: taniaribeiro_2@hotmail.com.
} 
informal. A forma clínica da tuberculose predominante foi pulmonar com pelo menos um agravo associado em que $34,5 \%$ obteve acompanhamento por Tratamento Diretamente Observado. Em Conhecimento, Atitude e Prática, os pacientes obtiveram níveis parecidos de adequação das respostas, sendo respectivamente, $66,3 \%, 66,2 \%$ e $70,4 \%$. CONCLUSÃO: foi possível identificar o perfil clínicoepidemiológico e sociodemográfico dos doentes em tratamento para tuberculose, bem como analisar os aspectos relacionados ao Conhecimento, Atitude e Prática frente à doença, contribuindo com a literatura científica e possibilita a criação, implementação e aperfeiçoamento de políticas públicas de saúde.

Palavras chave: Tuberculose. Pesquisas CAP. Saúde Pública.

ABSTRACT: OBJECTIVE: to know the epidemiological profile of patients undergoing tuberculosis treatment, as well as, their knowledge, attitude and practice towards the disease, evaluating aspects such as self-care and conduct. METHODS: cross-sectional descriptive and quantitative study carried out through a Knowledge, Attitude and Practice survey, in the city of Campina Grande-PB. The population consisted of patients diagnosed with tuberculosis between September 2017 and March 2018. Secondary data were obtained through the database of the Diseases Information and Notification System made available by the Department of Health of the municipality of Campina Grande-PB. Primary data were collected by form and their analysis comprised the values of distribution of absolute and relative frequencies, arithmetic means and obtaining epidemiological indicators. As for the concepts of Knowledge, Attitude and Practice, the theoretical and methodological framework of Marinho et al. RESULTS: male predominance, economically active age group, ethnicity / brown color, relatively low level of education, $34.5 \%$ were unemployed and $31 \%$ were working informally. The predominant clinical form of tuberculosis was pulmonary with at least one associated condition, in which $34.5 \%$ were followed up by Directly Observed Treatment. In Knowledge, Attitude and Practice, patients obtained similar levels of response adequacy, being, respectively, $66.3 \%, 66.2 \%$ and $70.4 \%$. CONCLUSION: it was possible to identify the clinical, epidemiological and sociodemographic profile of patients undergoing treatment for tuberculosis, as well as to analyze aspects related to Knowledge, Attitude and Practice in relation to the disease, contributing to the scientific literature and enabling the creation, implementation and improvement of policies public health.

Keywords: Tuberculosis. CAP Surveys. Public health. 


\section{INTRODUÇÃO}

A Tuberculose (TB) é uma doença causada pelo Mycobacterium tuberculosis e consiste na infecção que mais mata no mundo atualmente, embora seja evitável e curável por meio de medidas de prevenção e promoção à saúde (WHO, 2019). A mortalidade por TB também se encontra atrelada a fatores intrínsecos extrínsecos como o sistema imunológico do indivíduo e vulnerabilidades psicossociais e econômicas (BHARGAVA, BHARGAVA, 2020).

A associação da TB a outros agravos e comorbidades, como tabagismo, diabetes, HIV e outras doenças respiratórias, como a COVID-19 (doença infecciosa responsável pela pandemia do SARS-CoV-2), pode se tornar potencialmente letal ao paciente, além de se configurar como um problema de saúde pública de abrangência mundial e emergência em seu combate (ALAGNA, et al., 2020; BHARGAVA, BHARGAVA, 2020).

Presente em todas as regiões do globo, cerca de 10 milhões de pessoas adoeceram em decorrência da TB, assim como a doença ocasionou cerca de 1,5 milhões de óbitos em 2018. No Brasil, em 2019, foram diagnosticados 73.864 casos novos e 4.490 óbitos, o que equivale a um coeficiente de mortalidade de 2,2 óbitos/100 mil hab. Na Paraíba, que constitui um dos estados considerados prioritários para o controle da doença, foram notificados 1.107 casos novos no ano de 2019 , destes $82,6 \%$ consistiam na forma clínica pulmonar e, em 2018, obteve-se um coeficiente de mortalidade de 1,9 óbitos /100 mil hab. Além disso, o estado também apresentou percentuais de cura da TB pulmonar inferiores a $60 \%$, o que coloca a Paraíba abaixo da média nacional (71,9\%) (BRASIL, 2020).

De acordo com estudos, pacientes que apresentam mais conhecimento em relação ao processo saúde-doença possuem maior chance de adesão ao tratamento e menor abandono. É importante ressaltar que fatores como a falta de conhecimento, o meio no qual está inserido, o modo de viver e o próprio estigma social que cerca o indivíduo infectado com TB compreendem importantes barreiras 
para a adesão ao tratamento e alcance da cura (LUNA, et al., 2015; HOSSAIN, et al., 2015).

Acredita-se que o comportamento do indivíduo com relação ao enfrentamento da TB pode influenciar no êxito do tratamento. O comportamento compreende o que ele sabe, pensae faz a respeito da doença. E, pode ser avaliado através do Inquérito CAP (Conhecimento, Atitude e Prática), instrumento este que permite identificar características importantes de uma população no que tange ao conhecimento, atitude e prática diante de um problema (BRASIL, 2011; BRASIL, 2002; OLIVEIRA, NOGUEIRA, DE SÁ, 2015).

O inquérito CAP é uma avaliação formativa que além de identificar essas características em uma população, pode apontar caminhos para uma intervenção mais eficaz, pois, padrões comportamentais que afetam negativamente ou positivamente no enfrentamento da TB são descobertos e utilizados como ferramentas para o controle da doença (WHO,2018).

Nesse sentido, conhecer o perfil epidemiológico dos doentes em tratamento de TB, bem como, o conhecimento, atitude e prática dos mesmos frente à doença, avaliando assim, aspectos como autocuidado e condutas é de extrema importância para identificar problemas e necessidades que configuram barreiras para o enfrentamento da doença; e assim, contribuir com os profissionais e serviços de saúde para a construção de intervenções que promovam maior adesão do doente ao tratamento.

\section{METODOLOGIA}

Trata-se de um estudo transversal descritivo com abordagem quantitativa, a partir de um inquérito CAP realizado no município de Campina Grande-PB. A população do estudo foi composta pelos pacientes diagnosticados com tuberculose entre setembro de 2017 a março de 2018, maiores de 18 anos, residentes e em tratamento em Campina Grande. Foram identificados 53 casos de tuberculose, dos quais, foram excluídos doentes de tuberculose privados de liberdade, aqueles com 
incapacidade de compreensão e comunicação, sendoobtida uma amostra censitária de 29 pacientes.

A coleta de dados foi realizada através de entrevistas com os pacientes nos serviços de saúde e/ou no seu domicílio durante o período de janeiro a maio de 2018. A coleta dos dados secundários foi possibilitada por meio do banco de dados do Sistema de Informação de Agravos e Notificação disponibilizado pela Secretaria de Saúde do município de Campina Grande-PB. Para a coleta dos dados primários foi elaborado um formulário baseado no Guia para desenvolvimento de Pesquisa CAP da Organização Mundial de Saúde (2008) e no Manual do Aplicador do Inquérito CAP do Ministério da Saúde (MS) (2002), assim como estudos disponíveis na área (PENGPID, et al., 2016; MARINHO, 2002). O instrumento de coleta de dados consistiu em um formulário de entrevista com perguntas envolvendo a caracterização sociodemográfica, que permitiram caracterizar os pacientes; e uma segunda parte em que foram abordadas questões relacionadas à doença, contemplando o conhecimento da população em estudo, a atitude do paciente no enfrentamento da doença e por fim, as práticas diante do tratamento anti-TB.

A análise dos dados compreendeu os valores de distribuição de frequências absolutas e relativas, médias aritméticas e obtenção de indicadores epidemiológicos. Quanto aos conceitos de Conhecimento, Atitude e Prática, utilizou-se o referencial teórico-metodológico de Marinho et al. (2003). O conhecimento, atitude e prática adequados foram determinados segundo o estabelecido pelo MS, seguindo o padrão:

- Conhecimento adequado ( $\geq 66,6 \%$ de acertos): reconhecimento da TB como uma doença séria, dos sinais e sintomas da doença, sua causa, forma de transmissão, prevenção, tratamento, se tem cura, tomada de medicação, realização de exames e investigação decontatos/ familiares.

- Atitude adequada ( $\geq 66,6 \%$ de acertos): saber qual conduta tomar ao suspeitar de TB, a importância de seguir corretamente o esquema terapêutico, reconhecer o impacto que a doença causa no indivíduo, a importância da realização de exames de rotina (Baciloscopia de escarro, radiografias), de teste de HIV, do comparecimento às consultas, do relacionamento com a equipe, de se obter informações sobre a TB, de usar a máscara e de investigar contatos. 
- Prática adequada ( $\geq 66,6 \%$ de acertos): procurou o serviço de saúde quando apresenta os sintomas da doença, comparece às consultas mensais, toma a medicação corretamente, realiza exame de controle (Baciloscopia de escarro), utiliza/utilizou máscara, tem bom relacionamento com a equipe, foi realizada a investigação dos contatos.

O presente trabalho faz parte do Grupo de Pesquisa Avaliação de Serviços de Saúde cadastrado na UEPB-CNPq e foi coordenado e orientado por FIGUEIREDO, T. M. R. M., e foi realizado com apoio do PIBIC/CNPq-UEPB, cota 2017-2018, Conselho Nacional de Desenvolvimento Científico e Tecnológico - Brasil, em parceria com a Universidade Estadual da Paraíba, sendo submetido ao Comitê de Ética em Pesquisa da Universidade Estadual da Paraíba-CEP/UEPB, e aprovado segundo o parecer CAAE $n^{\circ}$ 67426617.2.0000.5187, atendendo às orientações inerentes ao protocolo de pesquisa contido na Resolução $n^{\circ} 466 / 12$ do Conselho Nacional de Saúde.

\section{RESULTADOS E DISCUSSÃO}

Os achados mostram o perfil epidemiológico dos sujeitos estudados com prevalência de vulnerabilidades sociais relacionadas à etnia/cor, ao nível de escolaridade e ao acesso a renda, dados estes compatíveis com outros estudos (BRASIL, 2019a; FUSCO, et al., 2017; PINTO, et al., 2017; BORGES, et al., 2018). Destaca-se predominância do sexo masculino $(72,4 \%)$, faixa etária economicamente ativa (idade média de 47 anos), etnia/cor parda (48,3\%), nível de escolaridade relativamente baixo (51,7\% cursaram até o ensino fundamental incompleto), $34,5 \%$ estavam desempregados e $31 \%$ exerciam trabalho informal.

Esta população é comumente mais afetada pela TB tendo em vista que indivíduos do sexo masculino possuem uma maior resistência quanto a procura dos serviços de saúde, bem como a execução de atividades laborais elevam os riscos para exposição à doença, além disso a baixa renda e escolaridade se encontram 
atreladas à falta de conhecimento acerca dapatologia, o que dificulta a adoção de medidas para prevenção e promoção à saúde (FONTES,et al., 2019).

A forma clínica da TB mais encontrada no estudo foi a pulmonar (82,8\%), a qual é a principal responsável pela cadeia de transmissão, outros estudos também demonstraram a prevalência dessa forma em detrimento das demais devido a predileção do bacilo pelo parênquima pulmonar (RAIMUNDO; GUIMARÃES; SILVA, 2016; PEREIRA, et al., 2015).

No que se refere aos agravos associados, a maior parte dos indivíduos relataram ter pelo menos um (01) agravo, sendo algumas dessas condições conhecidamente fatores facilitadores e/ou agravantes ao adoecimento por TB, como a Diabetes Mellitus, HIV e tabagismo, tais fatores podem gerar comprometimento ao sistema imunológico, o que dificulta a execução do tratamento, principalmente quanto à negativação bacteriológica, assim como aumenta as probabilidades para reações adversas aos antituberculostáticos e evidencia um predomínio de desfechos negativos, como abandono e óbito (SOARES, et al., 2020).

Quanto à modalidade de tratamento, o Ministério da Saúde (BRASIL, 2019b) recomenda que seja realizado o Tratamento Diretamente Observado (TDO), nesse estudo observou-se que $34,5 \%$ dos sujeitos entrevistados realizaram o tratamento supervisionado, sendo a média nacional para essa modalidade de 36,9\%, (BRASIL, 2019a).

Tabela 1. Características sociodemográficas e aspectos clínico-epidemiológicos dos sujeitos do estudo, Paraíba, Brasil, 2017-2018.

\begin{tabular}{|c|c|c|}
\hline \multicolumn{3}{|c|}{ CARACTERÍSTICAS SOCIODEMOGRÁFICAS } \\
\hline Variáveis & $\mathbf{N}[29]$ & $\%$ \\
\hline \multicolumn{3}{|c|}{ Sexo } \\
\hline Masculino & 21 & 72,4 \\
\hline Feminino & 8 & 27,6 \\
\hline \multicolumn{3}{|c|}{ Faixa etária } \\
\hline 18 a 29 anos & 4 & 13,8 \\
\hline 30 a 59 anos & 19 & 65,5 \\
\hline 60 ou mais & 6 & 20,7 \\
\hline
\end{tabular}




\begin{tabular}{|c|c|c|}
\hline \multicolumn{3}{|c|}{ Etnia/cor } \\
\hline Branca & 8 & 27,6 \\
\hline Negra & 7 & 24,1 \\
\hline Parda & 14 & 48,3 \\
\hline \multicolumn{3}{|c|}{ Escolaridade } \\
\hline Sem escolaridade declarada & 4 & 13,8 \\
\hline Ensino fundamental completo/incompleto & 11 & 37,9 \\
\hline Ensino médio completo/incompleto & 10 & 34,5 \\
\hline Ensino superior completo/incompleto & 4 & 13,8 \\
\hline \multicolumn{3}{|c|}{ Fonte de renda } \\
\hline Carteira assinada & 4 & 13,8 \\
\hline Renda informal & 9 & 31 \\
\hline Desempregado & 10 & 34,5 \\
\hline Outra & 6 & 20,7 \\
\hline \multicolumn{3}{|c|}{ Aspectos clínico-epidemiológicos } \\
\hline Variáveis & $\mathbf{N}[29]$ & $\%$ \\
\hline \multicolumn{3}{|c|}{ Forma clínica da TB } \\
\hline Pulmonar & 24 & 82,8 \\
\hline Extrapulmonar & 4 & 13,8 \\
\hline Pulmonar + Extrapulmonar & 1 & 3,4 \\
\hline \multicolumn{3}{|c|}{ Doenças e agravos } \\
\hline HIVIAIDS & 2 & 7 \\
\hline Etilismo & 3 & 10,3 \\
\hline Diabetes mellitus & 3 & 10,3 \\
\hline Hipertensão Arterial Sistêmica & 7 & 24,1 \\
\hline Uso de drogas & 1 & 3,4 \\
\hline Tabagista & 10 & 34,5 \\
\hline \multicolumn{3}{|c|}{ Modalidade do tratamento da TB } \\
\hline Autoadministrado & 18 & 62,1 \\
\hline TDO & 10 & 34,5 \\
\hline NS/NR & 1 & 3,4 \\
\hline
\end{tabular}

Fonte: dados da pesquisa, 2018.

Dos sujeitos entrevistados, $86,2 \%$ sabiam definir a seriedade da doença, $55,2 \%$ reconheceu de 1 a 3 sinais e sintomas provocados pela TB e $24,1 \%$ reconheceram mais de 3 sintomas, $44,8 \%$ identificaram a infecção como a causa etiológica da doença. Em relação à transmissão da doença, $72,4 \%$ soube relatar a forma correta de disseminação. Dos entrevistados, $65,5 \%$ não reconheceram como 
se previne a TB. Ao serem indagados sobre quem pode ser infectado pela TB, $82,7 \%$ respondeu que todos estão sujeitos.

Quanto ao tratamento da TB, 93,1\% relataram que o medicamento deve ser tomado diariamente, ao serem interrogados se a doença tem cura $100 \%$ afirmou que é curável, $100 \%$ também relatou que a pessoa pode se curar através da tomada dos medicamentos e acompanhamento em unidade de saúde, 48,2\% reconheceu o tratamento supervisionado como o mais indicado para se alcançar a cura, 65,5\% identificou de 1 a 2 exames que devem ser realizados durante o tratamento e $24,1 \%$ reconheceu todos os exames, e 55,1\% afirmou que o exame de escarro deve ser realizado mensalmente.

Outros estudos de Conhecimento, Atitude e Prática, revelaram também conhecimentos conflitantes em relação a tuberculose, havendo ainda baixo conhecimento em relação aos sintomas, a forma que a infecção se dá e maneiras de prevenção (HOSSAIN, et al., 2015; FREITAS, 2015). A carência de conhecimento pode desencadear estigma em relação a TB, bem como, atraso na procura do diagnóstico e tratamento, perpetuando a cadeia de transmissão e agravando o quadro de morbimortalidade (PENGPID, et al., 2016; BALOGUN, et al., 2019).

No que se refere à Atitude, predominou um posicionamento positivo quanto ao tratamento da TB, onde $86,3 \%$ afirmaram ser importante procurar o serviço de saúde de forma imediata, bem como tomar a medicação diariamente sem acompanhamento de leite ou outros alimentos na hora da ingestão do medicamento, realizar exames e consultas de rotina, ter um bom relacionamento com equipe de saúde, proporcionando um canal de vínculo e troca de conhecimentos e vivências.

Estudo de Inquérito CAP realizado em Timor-Leste, no sudeste asiático, revelou que os profissionais de saúde são de extrema importância na disseminação de informação acerca da TB, estando associados a uma boa Atitude que diz respeito a doença e ao seu aspecto estigmatizante (PENDPIG; PELTZER, 2019).

Sobre o impacto da TB na vida pessoal, 31\% relataram tristeza e 27,6\% sentiram medo. Houve também predominância de 93,1\% dos entrevistados que afirmaram que as pessoas têm preconceito com o doente de TB. O cenário do isolamento sociocultural e da privação econômica é um contexto que pode afetar negativamente os resultados por meio de atrasos no tratamento e/ou adesão 
inadequada ao tratamento, o que aumenta o risco de resistência aos medicamentos, da morbidade e da mortalidade. O estigma para o diagnóstico da TB em si também impede a busca de ajuda médica no início do curso da doença, resultando emcronicidade e maus resultados (SWEETLAND, et al., 2017).

No que tange os aspectos da Prática dos pacientes em relação ao tratamento e enfrentamento da tuberculose revelaram que $72,4 \%$ dos entrevistados disseram ter procurado o serviço de saúde imediatamente, sendo as Unidades Básicas de Saúde as mais procuradas pela população $(58,6 \%)$, estando de acordo com o que é previsto no Manual de Recomendações para o Controle da Tuberculose no Brasil (BRASIL, 2019b), que enfatiza que a Atenção Primária à Saúde (APS) deve ser o serviço de porta de entrada preferencial para o atendimento de diagnóstico da tuberculose, estando apta a dar prosseguimento ao tratamento e a orientação dos pacientes, bem como dos seus contatos.

Ao realizar a procura pelo serviço de saúde, em especial pela APS que se encontra inserida diretamente na comunidade a qual o usuário pertence, um vínculo com a equipe de saúde pode ser estabelecido de modo mais concreto, o que traz aspectos positivos ao tratamento da TB uma vez que o contato contínuo permite que sejam identificados os obstáculos para a execução do processo terapêutico, bem como concede a oportunidade de traçar metas e estratégias de saúde centradas na individualidade de cada usuário. Além disso, o serviço de saúde se torna um espaço para que o indivíduo obtenha mais conhecimento sobre sua doença, tornando-se mais ativo em relação ao seu processo saúde-doença, o que melhora consideravelmente as probabilidades de desfechos positivos ao tratamento (BERALDO, et al.,2017; FURLAN, et al., 2017).

Quanto às consultas de acompanhamento mensal para avaliação contínua, $86,2 \%$ afirmaram comparecer, além de tomar o medicamento diariamente com água, pela manhã e em jejum, indo ao encontro do que foi relatado nos itens de Conhecimento e Atitude.

Quanto à realização de exames de controle que incluem baciloscopia de escarro, radiografia e o teste HIV, 58,6\% realizaram um ou dois desses exames. $\mathrm{O}$ exame de escarro se encontra disponível na rede de atenção à saúde e constitui-se não somente como um exame padrão-ouro para o diagnóstico de casos bacilíferos 
da doença, mas, também, como um importante aliado durante o acompanhamento do tratamento para a tuberculose, pois a negativação bacteriológica ou a falta dela dá importantes indícios da evolução clínica do processo terapêutico, o que sinaliza a equipe de saúde sobre a necessidade de adequar o esquema medicamentoso, bem como facilita a detecção precoce de multirresistência bacteriana (GUIDONI, et al., 2015; LIMA, et al., 2016).

Em relação à busca ativa de contatos, $58,6 \%$ dos contatos foram examinados. A busca ativa de contatos e a realização dos exames preconizados pelo MS tornam-se grandes aliados no que diz respeito a quebra da cadeia de transmissão e na continuidade do cuidado do paciente de TB, tendo em vista que essas estratégias proporcionam o diagnóstico precoce, minimizando possíveis situações de coinfecção TB-HIV e/ou situações de droga-resistência e demais complicações no decorrer do tratamento (BRASIL, 2019b).

No que tange à utilização de máscara, $44,8 \%$ utilizaram no início do tratamento. De acordo com Brasil (2019b), o uso da máscara cirúrgica por sintomáticos respiratórios ou pacientes acometidos de TB pulmonar bacilífera é fundamental no serviço de saúde até a negativação da baciloscopia aliados a boa iluminação e ventilação, bem como a etiqueta respiratória, de modo a minimizar a proliferação do bacilo e reduzir a transmissão da doença.

Tabela 2. Conhecimento, Atitude e Prática dos indivíduos sobre Tuberculose, CampinaGrande- PB, Brasil, 2017-2018.

\begin{tabular}{|l|l|c|c|}
\hline \multicolumn{4}{|c|}{ NÍVEL DE CONHECIMENTO DOS INDIVÍDUOS SOBRE TB } \\
\begin{tabular}{|l|l|c|c|}
\hline Variáveis & N [29] & $\%$ \\
\hline \multicolumn{3}{|c|}{ Reconhecimento da TB como uma doença séria } \\
\hline Quão séria é a TB? & Um problema muito sério & 25 & 86,2 \\
\hline Quão séria é a TB em sua região? & Um problema muito sério & 13 & 44,8 \\
\hline $\begin{array}{l}\text { Reconhecimento de sinais e sintomas da doença, causa, forma de transmissão } \\
\text { e prevenção }\end{array}$ \\
\hline Quais os sinais e sintomas da TB? & Reconhece 1 a 3 sintomas. & 17 & 58,6 \\
\hline O que causa a TB? & Infecção. & 14 & 48,2 \\
\hline Como uma pessoa pode pegar TB? & $\begin{array}{l}\text { Reconhece O modo de } \\
\text { transmissão da TB. }\end{array}$ & 24 & 72,4 \\
\hline Quem pode ser infectado pela TB? & Todos. & 21 & 82,7 \\
\hline
\end{tabular}
\end{tabular}




\begin{tabular}{|c|c|c|}
\hline $\begin{array}{l}\text { Como uma pessoa pode prevenir de } \\
\text { adoecer por reconhece o modo de } \\
\text { prevenção da TB. }\end{array}$ & 19 & 65,5 \\
\hline \multicolumn{3}{|l|}{ Conhecimento adequado do tratamento e medicação } \\
\hline \begin{tabular}{|l|l|} 
Como é o tratamento da TB? & $\begin{array}{l}\text { Deve-se tomar os } \\
\text { medicamentos diariamente. }\end{array}$
\end{tabular} & 27 & 93,1 \\
\hline $\begin{array}{l}\begin{array}{l}\text { Qual o melhor turno para tomarDe manhã, em jejum. } \\
\text { medicação? }\end{array} \\
\end{array}$ & 28 & 96,5 \\
\hline $\begin{array}{l}\text { Qual a melhor forma de ingerir a Com água. } \\
\text { medicação? }\end{array}$ & 26 & 89,6 \\
\hline \multicolumn{3}{|l|}{ Reconhecimento sobre o processo de cura da TB } \\
\hline \begin{tabular}{|l|l|} 
A TB tem cura? & Sim. \\
\end{tabular} & 29 & 100 \\
\hline \begin{tabular}{|lr} 
Como a pessoa pode se curar da TB? & $\begin{array}{l}\text { Com medicamentos e } \\
\text { acompanhamento } \\
\text { unidade de saúde. }\end{array}$ \\
\end{tabular} & 29 & 100 \\
\hline $\begin{array}{l}\text { Qual o tratamento mais indicado para Supervisionado. } \\
\text { se alcançar a cura? }\end{array}$ & 14 & 48,2 \\
\hline \multicolumn{3}{|l|}{ Reconhecimento dos exames a serem feitos } \\
\hline \begin{tabular}{|l|l|} 
É necessário realizar quais exames & Reconhece 1 ou 2 exames. \\
durante otratamento da TB?
\end{tabular} & 19 & 65,5 \\
\hline $\begin{array}{l}\text { Com que frequência deve-se realizarMensalmente. } \\
\text { a coleta de escarro? }\end{array}$ & 16 & 55,1 \\
\hline \multicolumn{3}{|l|}{ Procura ao serviço de saúde e investigação de contatos } \\
\hline \begin{tabular}{|l|l|l} 
Qual serviço procurar primeiro se UBS. \\
suspeitar que está com TB?
\end{tabular} & 19 & 65,5 \\
\hline $\begin{array}{l}\text { Ao diagnosticar um paciente com TB, } \\
\text { é nim. } \\
\text { contatos/familiares? }\end{array}$ & 22 & 75,8 \\
\hline \multicolumn{3}{|c|}{$\begin{array}{l}\text { ATITUDE DOS INDIVÍDUOS COM TUBERCULOSE NO ENFRENTAMENTO DA } \\
\text { DOENÇA }\end{array}$} \\
\hline \begin{tabular}{|l|l} 
Variáveis &
\end{tabular} & $\mathbf{N}[29]$ & $\%$ \\
\hline \multicolumn{3}{|l|}{ Conduta a ser tomada ao suspeitar estar com TB } \\
\hline \begin{tabular}{|l|l|} 
O que tem que fazer se suspeitar|Procurar serviço de saúde \\
estar com TB?
\end{tabular} & 25 & 86,3 \\
\hline \multicolumn{3}{|l|}{ Esquema terapêutico } \\
\hline $\begin{array}{l}\text { É importante tomar a medicação Sim. } \\
\text { diariamente? }\end{array}$ & 29 & 100 \\
\hline $\begin{array}{l}\text { O horário de tomar a medicação Sim. } \\
\text { influencia no sucesso do tratamento? }\end{array}$ & 22 & 75,9 \\
\hline $\begin{array}{l}\text { Ingerir a medicação com leite ou Sim. } \\
\text { alimentos prejudica o tratamento? }\end{array}$ & 24 & 82,7 \\
\hline \multicolumn{3}{|l|}{ Impacto do diagnóstico da TB na vida do paciente } \\
\hline Como se sentiu ao saber que tem|Medo. & 8 & 27,6 \\
\hline
\end{tabular}




\begin{tabular}{|c|c|c|c|}
\hline \multirow{4}{*}{ TB? } & & & \\
\hline & Surpreso. & 4 & 13,7 \\
\hline & Envergonhado. & 5 & 17,4 \\
\hline & Triste. & 9 & 31,1 \\
\hline $\begin{array}{l}\text { As pessoas tem preconceito com } \\
\text { doentes de TB? }\end{array}$ & Sim. & 27 & 93,2 \\
\hline \multicolumn{4}{|c|}{ Realização de exames e consultas de rotina durante o tratamento } \\
\hline É importante fazer os exames? & Sim. & 28 & 96,6 \\
\hline $\begin{array}{l}\text { A coleta de escarro precisa ser feita } \\
\text { mensalmente? }\end{array}$ & Sim. & 18 & 62 \\
\hline $\begin{array}{l}\text { Todo paciente com TB deve fazer } \\
\text { exame para HIV? }\end{array}$ & Sim. & 19 & 65,6 \\
\hline $\begin{array}{l}\text { É importante ir mensalmente para as } \\
\text { consultas? }\end{array}$ & Sim. & 24 & 82,7 \\
\hline \multicolumn{4}{|c|}{ Relacionamento com a equipe de saúde } \\
\hline $\begin{array}{l}\text { É importante ter um bom } \\
\text { relacionamento/confiar na equipe de } \\
\text { saúde? }\end{array}$ & Sim & 29 & 100 \\
\hline \multicolumn{4}{|c|}{ Obtenção de informações sobre a TB } \\
\hline $\begin{array}{l}\text { Você acha que está bem orientado } \\
\text { (a) sobre a doença/tratamento? }\end{array}$ & Sim. & 26 & 89,7 \\
\hline $\begin{array}{l}\begin{array}{l}\text { Você acha que precisa de mais } \\
\text { informações } \\
\text { doença/tratamento? }\end{array} \\
\text { sobre }\end{array}$ & Sim. & 13 & 44,8 \\
\hline $\begin{array}{l}\text { Onde você acha mais fácil conseguir } \\
\text { informações sobre a TB? }\end{array}$ & $\begin{array}{l}\text { Com profissionais de } \\
\text { saúde. }\end{array}$ & 23 & 79,4 \\
\hline \multicolumn{4}{|c|}{ Uso de máscara e investigação de contatos } \\
\hline $\begin{array}{l}\text { Você acha que precisa usar máscara } \\
\text { sempre que sair em público? }\end{array}$ & Só no início do tratamento. & 16 & 55,2 \\
\hline $\begin{array}{l}\text { Você acha importante investigar se os } \\
\text { familiares também estão com TB? }\end{array}$ & Sim. & 24 & 82,7 \\
\hline \multicolumn{4}{|c|}{\begin{tabular}{|l|} 
Tratamento Supervisionado \\
\end{tabular}} \\
\hline $\begin{array}{l}\text { Você acha que o tratamento } \\
\text { supervisionado aumenta a chance de } \\
\text { cura? }\end{array}$ & Sim. & 20 & 68,9 \\
\hline \multicolumn{4}{|c|}{ PRÁTICA DO INDIVÍDUO NO ENFRENTAMENTO DA TUBERCULOSE } \\
\hline Variáveis & & $\mathbf{N}[29]$ & $\%$ \\
\hline \multicolumn{4}{|c|}{ Reação frente aos sintomas da TB } \\
\hline $\begin{array}{l}\text { O que você fez ao apresentar os } \\
\text { sintomas da TB? }\end{array}$ & $\begin{array}{l}\text { Procurou serviço de saúde } \\
\text { imediatamente. }\end{array}$ & 21 & 72,4 \\
\hline $\begin{array}{l}\text { Ao buscar um serviço de saúde| } \\
\text { devido seus sintomas, qual serviço } \\
\text { buscou primeiro? }\end{array}$ & UBS. & 17 & 58,6 \\
\hline
\end{tabular}




\begin{tabular}{|c|c|c|}
\hline $\begin{array}{l}\begin{array}{l}\text { Quando tem dúvidas, onde procura } \\
\text { informações primeiro? }\end{array} \\
\text { saúde. }\end{array}$ & 22 & 75,9 \\
\hline \multicolumn{3}{|l|}{ Comparecimento às consultas mensais } \\
\hline $\begin{array}{l}\text { Você vai mensalmente para as } \\
\text { consultas? }\end{array}$ & 25 & 86,2 \\
\hline \multicolumn{3}{|l|}{ Tomada de medicamentos anti-TB } \\
\hline $\begin{array}{l}\text { Você toma os medicamentos Sim. } \\
\text { diariamente? }\end{array}$ & 28 & 96,5 \\
\hline \begin{tabular}{|l|l} 
Como você toma a medicação? & Com água. \\
\end{tabular} & 29 & 100 \\
\hline Que horário você toma a medicação? De manhã, em jejum. & 28 & 96,5 \\
\hline \multicolumn{3}{|c|}{ Realização de exames de controle (baciloscopia de escarro, raio-x, HIV) } \\
\hline $\begin{array}{l}\text { Você fez quais exames durante o|Fez } 1 \text { ou } 2 \text { exames. } \\
\text { tratamento da TB? }\end{array}$ & 17 & 58,6 \\
\hline $\begin{array}{l}\text { Você faz o exame de escarrosim. } \\
\text { mensalmente? }\end{array}$ & 10 & 34,5 \\
\hline \multicolumn{3}{|l|}{ Utilização da máscara } \\
\hline Você fez/faz o uso da máscara|Só no início do tratamento & 13 & 44,8 \\
\hline durante o tratamento? $\quad$ Nunca & 13 & 44,8 \\
\hline \multicolumn{3}{|l|}{ Relacionamento com a equipe de saúde } \\
\hline $\begin{array}{l}\text { Você confia/tem um bom Sim. } \\
\text { relacionamento com a equipe de } \\
\text { saúde que o assiste? }\end{array}$ & 27 & 93,1 \\
\hline \multicolumn{3}{|l|}{ Investigação de contatos } \\
\hline Seus $\quad$ familiares/contatos foram Sim. & 12 & 41,4 \\
\hline examinados após seu diagnóstico? Não & 17 & 58,6 \\
\hline
\end{tabular}

Fonte: dados da pesquisa, 2018.

Constatou-se que, no que tange ao Conhecimento, Atitude e Prática, os pacientes tiveram níveis equiparados de adequação das respostas. O item Prática foi o único que obteve pontuação acima do valor considerado adequado ( $\geq 66,6 \%$ ), podendo estar associado a ações de educação permanente, bem como do vínculo estabelecido pelos profissionais de saúde no sentido de conscientização dessa população.

Estudo realizado na Etiópia (DATIKO, et al., 2019) reforça que o nível socioeconômico, acesso à educação e a serviços de saúde são fatores chaves no que diz respeito a aquisição de conhecimento, melhora da percepção e, consequente, melhor prática em relação à tuberculose. A ausência de fatores 
facilitadores, como esses, acarreta no aumento do estigma, o que está intrinsecamente associado a um impacto mais negativo da doença.

Mesmo com os melhores valores obtidos na prática, o TDO é uma estratégia com inúmeros benefícios, mas no estudo em questão foi pouco explorada, principalmente diante deum grupo com tantas vulnerabilidades que podem inferir em desfechos desfavoráveis. Sua implementação apresenta várias limitações que podem ser superadas com práticas flexíveis e capacitação dos recursos humanos envolvidos (RUSSONI; TRINDADE, 2019).

Gráfico 1. Conhecimento, Atitude e Prática adequados sobre tuberculose de acordo com asrespostas dos sujeitos da pesquisa, Campina Grande- PB, Brasil, 2017-2018. Fonte: dados da pesquisa, 2018.

\section{Conhecimento, Atitude e Prática adequados}

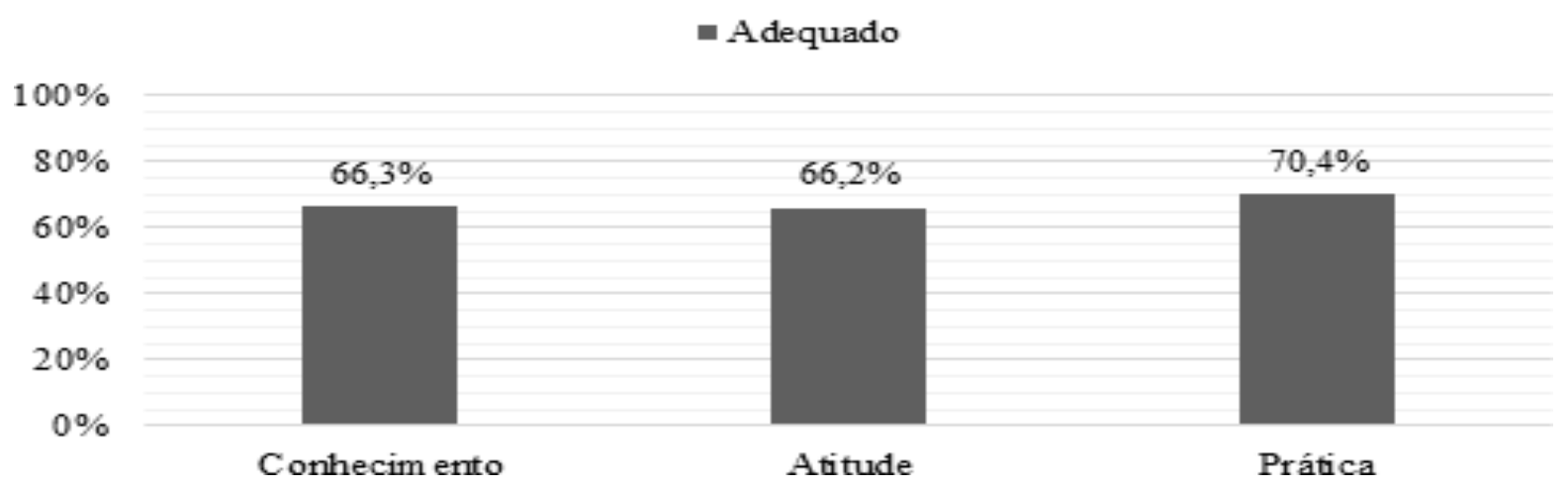

\section{CONCLUSÃO}

Ao serem avaliados os aspectos ligados ao Conhecimento, Atitude e Prática, constatou- se que há lacunas e isto repercute na necessidade de direcionar ações educativas baseadas nas reais necessidades desse público, erradicando possíveis falhas à promoção da saúde e fundamentando adoção de práticas mais favoráveis e adequadas ao contexto em que esses indivíduos estão inseridos.

Espera-se que os achados desse estudo possam fortalecer a criação, implementação e aperfeiçoamento de políticas públicas de saúde, por meio da 
compreensão do contexto epidemiológico e percepção dos indivíduos acerca da doença, a fim de facilitar a identificação de fatores favoráveis e desfavoráveis no combate à tuberculose. Outrossim, o trabalho busca contribuir com a divulgação e aprimoramento de conhecimento científico por meio de pesquisas com base em Inquéritos de Conhecimento, Atitude e Prática.

Tendo em vista a dificuldade no acesso ao banco de dados e à população durante a fase de coleta, o estudo obteve uma amostra reduzida, o que ocasionou limitações quanto ao desenvolvimento de dados com caráter mais abrangente em relação aos sujeitos do estudo.

\section{REFERÊNCIAS BIBLIOGRÁFICAS}

ALAGNA, R., et al. Celebrating TB day at the time of COVID-19. European Respiratory Journal, $\quad$ v. $55, \quad$ n. $4, \quad 2020 . \quad$ Disponível $\quad$ em: https://www.ncbi.nlm.nih.gov/pmc/articles/PMC7113797/. Acesso em: 11 de maio de 2020.

BALOGUN, M. R., et al. Predictors of tuberculosis knowledge, attitudes and practices in urban slums in Nigeria: a cross-sectional study. Pan African Medical Journal, v. 32, n. 60, 2019. Disponivel em: https://www.ncbi.nlm.nih.gov/pmc/articles/PMC6560972/pdf/PAMJ- 32-60.pdf . Acesso em: 13 de abril de 2020.

BERALDO, A.A., et al. Adesão ao tratamento da tuberculose na Atenção Básica: percepção de doentes e profissionais em município de grande porte. Revista Escola AnnaNery, v. 21, n. 4, p. 1-8, 2017. Disponível em: https://www.redalyc.org/pdf/1277/127752022025.pdf. Acesso em: 18 de janeiro de 2021.

BHARGAVA, A., BHARGAVA, M. Tuberculosis deaths are predictable and preventable: Comprehensive assessment and clinical care is the key. Journal of Clinical Tuberculosis, v. 19, 2020. Disponível em: https://www.ncbi.nlm.nih.gov/pmc/articles/PMC7082610/pdf/main.pdf. Acesso em: 11 de maio de 2020.

BORGES, R. M., et al. Perfil epidemiológico da tuberculose nas macrorregiões de saúde do estado de Minas Gerais no período de 2006 a 2016. HU Revista, v. 44, n. 3, p. 333-341, 2018.

Disponivel em: http://docs.bvsalud.org/biblioref/2020/01/1048094/14034-manuscrito-semidentificacao-dos-autores-106833-1-10-20190621.pdf. Acesso em: 02 de abril de 2020.

BRASIL. Ministério da Saúde. Secretaria de Vigilância em Saúde. Boletim epidemiológico Tuberculose 2020, n. especial, 2020. Disponível em: http://www.aids.gov.br/ptbr/pub/2020/boletim-epidemiologico-de-turbeculose-2020. Acesso em: 31 de janeiro de 2021.

BRASIL. Ministério da Saúde. Secretaria de Vigilância em Saúde. Boletim epidemiológico Brasil livre da tuberculose: evolução dos cenários epidemiológicos e operacionais da doença. Brasília: Ministério da Saúde, v.50, n.09, 2019a. Disponível em: http://portalarquivos2.saude.gov.br/images/pdf/2019/marco/22/2019-009.pdf. Acesso em: 03 de março de 2020. 
BRASIL. Ministério da Saúde. Secretaria de Vigilância em Saúde. Departamento de Vigilância das Doenças Transmissíveis. Manual de Recomendações para o Controle da Tuberculose no Brasil. Brasília: Ministério da Saúde, 2 ed, 2019b. Disponível em: http://portalarquivos2.saude.gov.br/images/pdf/2019/marco/28/manual-recomendacoes.pdf. Acesso em: 03 de março de 2020.

BRASIL. Ministério da educação. Instituto Nacional do Desenvolvimento da Educação. Manual do Aplicador do Estudo CAP, 2002. Disponível em: https://www.fen.ufg.br/fen_revista/v14/n1/pdf/v14n1a19.pdf. Acesso em: 03 de maio de2017.

BRASIL. Ministério da Saúde. Secretaria de Vigilância em Saúde. Departamento de Vigilância Epidemiológica. Tratamento diretamente observado (TDO) da tuberculose na atenção básica: protocolo de enfermagem. Brasília: Ministério da Saúde, 2011. Disponível em: http://bvsms.saude.gov.br/bvs/publicacoes/tratamento_diretamente_observado_tuberculose.pdf. Acesso em: 03 de março de 2020.

DATIKO, D. G., et al. Knowledge, attitudes, and practices related to TB among the general population of Ethiopia: Findings from a national cross-sectional survey. PLoS ONE, v. 14, n.10, 2019. Disponível em: https://journals.plos.org/plosone/article/file?id=10.1371/journal.pone.0224196\&type=printable. Acesso em:13 de abril de 2020.

FREITAS, I. M., et al. Fatores associados ao conhecimento sobre tuberculose e atitudes das famílias de pacientes com a doença em Ribeirão Preto, São Paulo. Revista Brasileira de Epidemiologia, v. 18, n. 2, p. 326-340, 2015. Disponível em: https://www.scielo.br/scielo.php?pid=S1415- 790X2015000200326\&script=sci_arttext\&tlng=pt . Acesso em: 13 de abril de 2020.

FONTES, G.J.F., et al. Perfil epidemiológico da tuberculose no Brasil no período de 2012 a 2016. Revista Brasileira de Educação e Saúde, v. 9, n. 1, p. 19-26, 2019. Disponível em: https://editoraverde.org/gvaa.com.br/revista/index.php/REBES/article/view/6376. Acesso em: 14 de janeiro de 2021.

FURLAN, M.C.R., et al. O vínculo com o profissional de saúde no tratamento de tuberculose: percepção dos usuários. Revista de Enfermagem do Centro-Oeste Mineiro, v. 7, e. 1934, p. 1-12, 2017. Disponível em: http://seer.ufsj.edu.br/index.php/recom/article/view/1934. Acesso em: 18 de janeiro de 2021.

FUSCO, A. P. B. et al. Spatial distribution of tuberculosis in a municipality in the interior of São Paulo, 2008-2013. Revista Latino-Americana Enfermagem, v. 17, n. 25, e. 2888, 2019. Disponível em: https://www.scielo.br/scielo.php?script=sci_arttext\&pid=S010411692017000100340. Acesso em: 02 de abril de 2020.

GUIDONI, L.M., et al. Fatores que interferem na qualidade das amostras e dos procedimentos de coleta de escarro em sintomáticos respiratórios. Revista Brasileira de Pesquisa em Saúde, v. 17, n. 2, p. 61-69, 2015. Disponível em: https://www.periodicos.ufes.br/rbps/article/download/13189/9239. Acesso em: 18 de janeiro de 2021.

HOSSAIN, S., et al. Factors associated with poor Knowledge among adults on tuberculosis in Bangladesh: results from a nationwide survey. Journal of Health, Populacion and Nutrition, $v$. 34, n. 2, 2015. Disponivel em: https://jhpn.biomedcentral.com/articles/10.1186/s41043-0150002-4. Acesso em: 03 de maio de 2017.

LIMA, L.M., et al. Avaliação do acompanhamento e desfecho de casos de tuberculose em município do sul do Brasil. Revista Gaúcha de Enfermagem, v. 37, n. 1, p. 1-7, 2016. Disponivel em: https://www.scielo.br/pdf/rgenf/v37n1/0102-6933-rgenf-37-1-1983- 
144720160151467.pdf. Acesso em: 18 de janeiro de 2021.

LUNA, F. D. T. et al. Adherence to Tuberculosis Treatment: Programatic Vulnerability Elements. International Archives de Medicine, v. 8 n. 207, 2015. Disponível em http://imed.pub/ojs/index.php/iam/article/view/1239/990. Acesso em: 03 de maio de 2017.

MARINHO, L. A. B. et al. Conhecimento, atitude e prática do auto- exame das mamas em centros de saúde. Revista Saúde Pública, v.37, n.5, p. 576- 582. Disponível em: http://www.scielo.br/pdf/rsp/v37n5/17471.pdf. Acesso em: 03 de março de 2020.

OLIVEIRA, L. C. S.; NOGUEIRA, J. A.; DE SÁ, L. D. A discursividade do sujeito sobre sentimentos associados ao enfrentamento da tuberculose. Revista Eletrônica de Enfermagem, v.17, n.1, p. 12-20, 2015. Disponível em: https://revistas.ufg.br/fen/article/view/24523. Acesso em: 03 de maio de 2017.

PENGPID, S., et al. Knowledge, attitudes, and practices about tuberculosis and choice of communication channels in Thailand. Journal of Infection in Developing Countries, v. 10, n. 7, p. 694-703, 2016. Disponivel em: https://jidc.org/index.php/journal/article/view/27482800/1540. Acesso em: 13 de abril de 2020.

PENDPIG, S., PELTZER, K. Knowledge, Attitudes, and Practices Regarding Tuberculosis in Timor-Leste: Results From the Demographic and Health Survey. Journal of Preventive Medicine \& Public Health, v. 52, p. 115-122, 2019. Disponível em: https://www.ncbi.nlm.nih.gov/pmc/articles/PMC6459764/pdf/jpmph-52-2-115.pdf. Acesso em: 20 de maio de 2020.

PEREIRA, J. C., et al. Perfil e seguimento dos pacientes com tuberculose em município prioritário no Brasil. Revista de Saúde Pública, v. 49, n. 6, 2015. Disponível em: https://www.scielo.br/pdf/rsp/v49/pt_0034-8910-rsp-S0034-89102015049005304.pdf. Acessoem: 02 de abril de 2020.

PINTO, P. F. P. S., et al. Perfil epidemiológico da tuberculose no município de São Paulo de 2006 a 2013. Revista Brasileira de Epidemiologia, v. 20, n. 3, p. 549-557, 2017. Disponível em: $\quad$ https://www.scielo.br/scielo.php?pid=S1415790X2017000300549\&script=sci_abstract\&tlng=pt. Acesso em: 02 de abril de 2020.

RAIMUNDO, A. G.; GUIMARÃES, A. M. D. N.; SILVA, S. C. P. S. Tuberculose: o perfil do novo milênio. Revista de Enfermagem UFPE On line, v. 10, n. 3, p. 1387-1396, 2016. Disponível em: https://periodicos.ufpe.br/revistas/revistaenfermagem/article/viewFile/11079/12519. Acesso em: 02 de abril de 2020.

RUSSONI, B.; TRINDADE, A. A. M. Estratégia de Tratamento Diretamente Observado (DOTS) para Tuberculose no Brasil: um estudo qualitativo. Revista Saúde Coletiva UEFS, v. 9, p. 7078, 2019.2 Disponível em: http://periodicos.uefs.br/index.php/saudecoletiva/article/view/4060/3804. Acesso em: 07 de janeiro de 2021.

SOARES, L.N., et al. Relação entre multimorbidade e o desfecho do tratamento da tuberculose pulmonar. Revista Gaúcha de Enfermagem, v. 41, p. 1 - 9, 2020. Disponível em: https://www.seer.ufrgs.br/RevistaGauchadeEnfermagem/article/download/108154/58695.

Acesso em: 14 de janeiro de 2021.

SWEETLAND, A. C., et al. Addressing the tuberculosis-depression syndemic to end the tuberculosis epidemic. International Journal of Tuberculosis and Lung Disease, v. 21, n.8, p. 852-861, $2017 . \quad$ Disponível em https://www.ncbi.nlm.nih.gov/pmc/articles/PMC5759333/pdf/nihms931556.pdf. Acesso em:13 de abril de 2020. 
WORLD HEALTH ORGANIZATION. Cataloguing-in-Publication Data Advocacy, communication and social mobilization for TB control: a guide to developing knowledge, atitude and practice surveys. 2008. Disponível em:

http://apps.who.int/iris/bitstream/handle/10665/43790/9789241596176_eng.pdf;jsessionid=E0 38CB0E793854A22B3357B69E167DB1?sequence=1. Acesso em: 17 de maio de 2020.

WORLD HEALT ORGANIZATION (WHO). Global tuberculosis report 2018. Geneva: World Organization. 2018. Disponível em: https://apps.who.int/iris/bitstream/handle/10665/274453/9789241565646eng.pdf?sequence=1\&isAllowed=y. Acesso em: 03 de março de 2020.

WORLD HEALT ORGANIZATION (WHO). Global tuberculosis report 2019. Geneva: World Health Organization. $2019 . \quad$ Disponivel $\quad$ em: https://apps.who.int/iris/bitstream/handle/10665/329368/9789241565714-eng.pdf?ua=1. Acesso em: 03 de março de 2020. 